\title{
Evaluation of a National Call Center and a Local Alerts System for Detection of New Cases of Ebola Virus Disease — Guinea, 2014-2015
}

\author{
Christopher T. Lee, MD 1,2,3; Marc Bulterys, MD, PhD²,4,5; Lise D. Martel, PhD²,6; Benjamin A. Dahl PhD 2,5
}

The epidemic of Ebola virus disease (Ebola) in West Africa began in Guinea in late 2013 (1), and on August 8, 2014, the World Health Organization (WHO) declared the epidemic a Public Health Emergency of International Concern (2). Guinea was declared Ebola-free on December 29, 2015, and is under a 90 day period of enhanced surveillance, following 3,351 confirmed and 453 probable cases of Ebola and 2,536 deaths (3). Passive surveillance for Ebola in Guinea has been conducted principally through the use of a telephone alert system. Community members and health facilities report deaths and suspected Ebola cases to local alert numbers operated by prefecture health departments or to a national tollfree call center. The national call center additionally functions as a source of public health information by responding to questions from the public about Ebola. To evaluate the sensitivity of the two systems and compare the sensitivity of the national call center with the local alerts system, the CDC country team performed probabilistic record linkage of the combined prefecture alerts database, as well as the national call center database, with the national viral hemorrhagic fever (VHF) database; the VHF database contains records of all known confirmed Ebola cases. Among 17,309 alert calls analyzed from the national call center, 71 were linked to 1,838 confirmed Ebola cases in the VHF database, yielding a sensitivity of $3.9 \%$. The sensitivity of the national call center was highest in the capital city of Conakry (11.4\%) and lower in other prefectures. In comparison, the local alerts system had a sensitivity of $51.1 \%$. Local public health infrastructure plays an important role in surveillance in an epidemic setting.

Passive surveillance for Ebola in Guinea consists of telephone calls from health centers and community members (alert calls) to report community deaths and symptomatic patients. Early in the response, all alerts were reported directly to local prefectures* and were investigated by a prefecture health department surveillance team to determine whether the patient met the suspected Ebola case definition, or whether the reported death occurred in a person who was at high risk for Ebola (4). In November 2014, the Government of Guinea, with funding from the CDC Foundation, established the national toll-free call center as a single point of contact to

\footnotetext{
${ }^{*}$ Guinea is divided into 34 prefectures; each prefecture has a public health department. Prefectures are further divided into sous-prefectures, except in the case of the capital city of Conakry, which is divided into five communes.
}

facilitate alert reporting but kept the local alert lines in place. Calls to the national call center are received by operators who enter alert information into a database before routing the call to a dispatch team who informs the local prefecture. Prefectures are therefore notified of all alerts regardless of the source of the call and investigate all alerts originating within the prefecture. Clinical specimens are collected from suspected cases and community deaths for Ebola testing.

The VHF database contains data on all persons who were tested for Ebola and all known, confirmed Ebola cases. Neither the national call center database nor the local alerts system contains identifiers shared with the VHF database. To compare the sensitivity of the national call center and local alerts system to detect new Ebola cases using the VHF database, probabilistic record linkage (a method that calculates the probability that two records refer to the same entity) was used to determine whether confirmed Ebola cases in the VHF database were linked to local or national alert calls.

During November 5, 2014-August 31, 2015, a total of 185,437 unique calls to the national call center, including $22,660(12 \%)$ alert calls, were analyzed; the other 162,777 $(88 \%)$ calls were primarily requests for public health information. Among the alert calls, 5,351 (24\%) were excluded because identifier data were missing, leaving 17,309 for analysis. These call center records were linked to 19,074 records in the VHF database for the same time period (excluding 311 records with missing identifiers) to measure call center sensitivity for detecting confirmed cases.

Fields in the databases for the local prefecture alerts system were standardized nationwide beginning April 1, 2015. To calculate the sensitivity of the local alerts system, records of 8,667 calls received during April 1, 2015-August 31, 2015, from four prefectures with active Ebola cases during that time period (Conakry, Coyah, Dubréka, and Forécariah) were merged into a data set for linkage with 9,454 VHF records from the same prefectures and time period.

Variables in all data sets (first name, surname, age, sex, village, sous-prefecture, and prefecture) were standardized to string variables with Soundex transformation of proper names. Soundex is a phonetic algorithm for indexing names by sound and has been used to perform accurate record linkage while preserving patient confidentiality (5). The data sets were matched by means of a probabilistic record linkage 
algorithm (G) using statistical software. Probabilistic record linkage uses a measure of the similarity between string variables (Levenshtein distance) to find matches between data sets using identifiers that might be spelled slightly differently when shared unique identifiers do not exist. Validation of matches was performed by drawing a random sample of 200 matched pairs and manually confirming actual matches. The manual confirmation process generated a receiver operating characteristic (ROC) curve, which plotted the sensitivity and specificity of actual matching for each match probability score produced by the algorithm. A match probability score of 0.80 was defined as the cutoff value on the ROC curve with equal sensitivity and specificity for actual matches (75\%). For each system, sensitivity was calculated as the proportion of confirmed cases in the VHF database with a match found within either the national call center database or within the prefecture alerts database. Validation of the sensitivity estimate from the national call center-VHF linkage was performed by: 1) drawing two additional random subsamples of 200 confirmed cases from the VHF database; and 2) manually confirming the matches identified by the probabilistic record linkage algorithm to determine the proportion of confirmed cases identified as a result of their being reported to the national call center. Sensitivity of the local alerts system was determined by manual confirmation of matches for all confirmed cases in the VHF from the same prefectures and time period.

During the study period, the number of daily alert calls to the national call center remained stable, the number of local alerts increased, and the number of confirmed Ebola cases declined (Figure). Linkage resulted in 1,778 matches between the national call center and VHF databases, 71 of which were confirmed cases. During the same period, there were 1,838 confirmed Ebola cases, with a resulting sensitivity (proportion of confirmed cases in the national call center database) of $3.9 \%$ for the call center (Table 1). Two random subsamples of 200 confirmed cases in the VHF database were drawn for validation purposes, with matches manually verified. Both subsamples contained 12 exact matches between databases, with a sensitivity estimate of $6.0 \%$.

FIGURE. Calls reporting community deaths and suspected Ebola virus disease (Ebola) cases from the national call center and local prefectures compared with confirmed Ebola cases in the viral hemorrhagic fever (VHF) database - Guinea, November 5, 2014-August 31, 2015

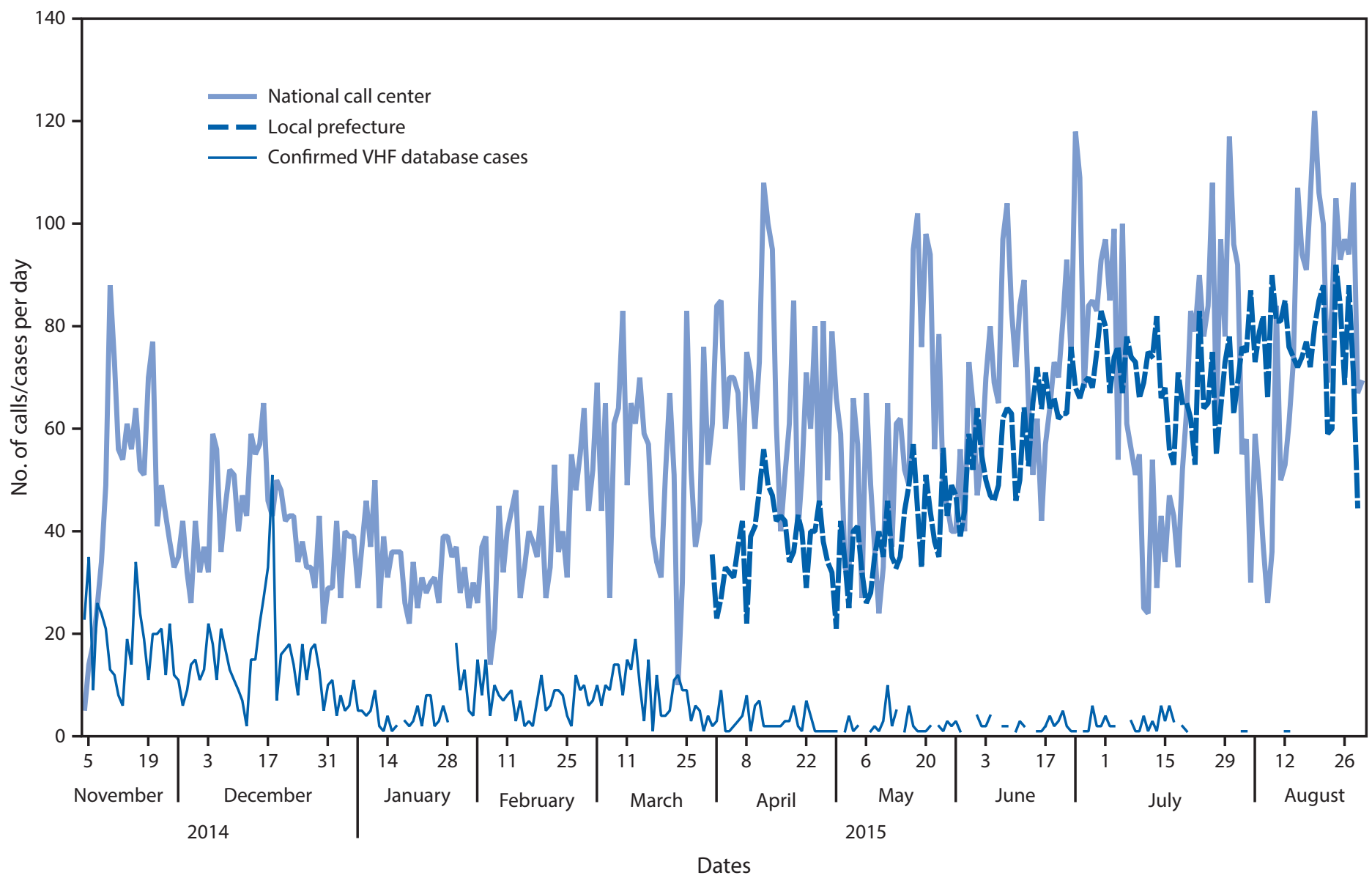


TABLE 1. Sensitivity of calls to the national call center and to local prefectures - Guinea, November 2014-August 2015

\begin{tabular}{lcr}
\hline Source & $\begin{array}{c}\text { Cases detected/ } \\
\text { confirmed VHF } \\
\text { database cases }\end{array}$ & $\begin{array}{l}\text { Sensitivity } \\
\%(95 \% \mathrm{Cl})\end{array}$ \\
\hline National call center* $(\mathrm{n}=\mathbf{1 7 , 3 0 9}$ alerts) & $71 / 1,838$ & $3.9(3.0-4.9)$ \\
Validation subsample 1 & $12 / 200$ & $6.0(3.1-10.3)$ \\
Validation subsample 2 & $12 / 200$ & $6.0(3.1-10.3)$ \\
Alert database (active prefectures) $)^{\dagger, \S}$ & $120 / 221$ & $54.3(47.8-70.0)$ \\
$\quad(n=8,667$ alerts) & $113 / 221$ & $51.1(44.3-57.9)$ \\
Local source $(\mathrm{n}=7,038$ alerts) & $7 / 221$ & $3.2(1.3-6.4)$ \\
National source $(\mathrm{n}=1,629$ alerts) & &
\end{tabular}

Abbreviations: $\mathrm{Cl}=$ confidence interval; $\mathrm{VHF}=$ viral hemorrhagic fever.

* November 2014-August 2015.

† Active prefectures (Dubréka, Conakry, Coyah, and Forécariah) are those with active Ebola cases during the period since the prefecture alert databases were standardized on April 1, 2015; data before this date are incomplete and inconsistent across prefectures.

$\S$ April 2015-August 2015.

Linkage between the local alerts database and VHF database identified 5,006 matches, 120 of which were confirmed cases. Among these, 113 originated locally and seven were first reported to the national call center. There were 221 confirmed cases in the VHF database in the same prefectures and time frame, resulting in a sensitivity estimate of $51.1 \%$ for local alert calls and $3.2 \%$ for the national call center.

Sensitivity estimates were calculated by prefecture (Table 2). Sensitivity of the national call center was highest in Conakry $(11.4 \%)$ and lower in other prefectures; there were 13 prefectures with confirmed Ebola cases where the sensitivity of the call center was $<1 \%$. Analysis of the local alerts database indicated varying patterns of sensitivity of local alerts; sensitivity was highest in Dubréka (79.3\%) and lowest in Conakry (30.2\%). Analysis of the local alerts database also demonstrated that sensitivity of the national call center was lower than the local alerts system in each of the active prefectures $^{\dagger}$ studied (Table 2).

\section{Discussion}

Sensitive surveillance mechanisms are critical for detecting outbreaks early and reducing transmission in an epidemic setting (7). In Guinea, passive surveillance detected approximately half of cases in active prefectures during the study period; the remainder were detected either by Ebola treatment units or through tracing contacts of known cases. The majority of calls that resulted in identification of confirmed cases of Ebola originated from calls to local prefectures. The sensitivity of both the national call center and local alerts systems varied by prefecture; however, for all prefectures studied, local alerts were more sensitive than the call center.

\footnotetext{
${ }^{\dagger}$ Active prefectures (Dubréka, Conakry, Coyah, and Forécariah) are those with active Ebola cases during the period since the prefecture alerts databases were standardized (on April 1, 2015); data before this date are incomplete and inconsistent across prefectures.
}

\section{Summary}

What is already known about this topic?

Little is known about the sensitivity of call centers for Ebola case-finding in an epidemic setting.

What is added by this report?

During the Ebola epidemic in Guinea, approximately half of cases were reported as alert calls. The sensitivity of passive surveillance systems can be compared using probabilistic record linkage. Calls to prefecture health departments were more sensitive for case detection than those to a national call center in all prefectures studied.

What are the implications for public health practice?

Although the national call center provided public health information for a high volume of calls, its low sensitivity for Ebola case detection limits its utility as a surveillance system. Prefecture health departments play a key role in surveillance and should be supported.

The findings in this report are subject to at least three limitations. First, data quality issues in the call center database resulted in a high volume of calls being excluded from analysis, which might have resulted in a lower or higher sensitivity estimate. Second, the local alerts databases were standardized later in the response than the national call center database, and at a time when few prefectures had active Ebola cases. Finally, mismatches resulting from the probabilistic record linkage of the national call center database with the VHF might have affected the accuracy of sensitivity estimates; based on the ROC curve, the sensitivity and specificity of matching was known to be $75 \%$. Despite these limitations, the sensitivity estimates for the national call center were nearly identical using two validation steps. Random subsamples with manual validation of the national call center sensitivity matches provided internal validation of the matching procedure. Estimates from the local alerts database provided external validation of those estimates generated from the national call center database.

Given the high call volume recorded in Guinea and the low sensitivity for identification of cases, the national call center was likely to have been more valuable in providing public health information than in case detection. Although nationwide call centers were established in response to the Ebola epidemic in Guinea, Liberia, and Sierra Leone, the sensitivity of those call centers for Ebola detection has not yet been studied. In Sierra Leone, a study of the nationwide call center found that alert calls resulted in same- or next-day field responses to $81 \%$ of deaths but only $45 \%$ of possible cases, highlighting the need to scale up local response services (8). These findings underscore the limited sensitivity of the national call center in Guinea and the importance of local public health infrastructure for Ebola surveillance. 
TABLE 2. Sensitivity estimates by prefecture for national call center alerts and local alerts in 12 prefectures* — Guinea, November $2014-$ August 2015

\begin{tabular}{|c|c|c|c|c|c|}
\hline \multirow[b]{3}{*}{ Prefecture } & \multicolumn{5}{|c|}{ Alert source } \\
\hline & \multicolumn{2}{|c|}{ National call center database ${ }^{\dagger}$} & \multicolumn{3}{|c|}{ Local database ${ }^{\S}$ (active prefectures ${ }^{\natural}$ ) } \\
\hline & $\begin{array}{l}\text { Confirmed VHF } \\
\text { database cases }\end{array}$ & $\begin{array}{c}\text { National call center } \\
\text { matches [sensitivity (\%)] }\end{array}$ & $\begin{array}{l}\text { Confirmed VHF } \\
\text { database cases }\end{array}$ & $\begin{array}{c}\text { Local prefecture } \\
\text { matches [sensitivity (\%)] }\end{array}$ & $\begin{array}{c}\text { National call center } \\
\text { matches [sensitivity (\%)] }\end{array}$ \\
\hline Boké & 32 & $1(3.1)$ & NA & NA & NA \\
\hline Conakry & 343 & 39 (11.4) & 53 & $16(30.2)$ & $6(11.3)$ \\
\hline Coyah & 184 & $3(1.6)$ & 8 & $5(62.5)$ & $0(0.0)$ \\
\hline Dubréka & 122 & $1(0.8)$ & 29 & $23(79.3)$ & $0(0.0)$ \\
\hline Forécariah & 411 & $13(3.2)$ & 131 & $69(52.7)$ & $1(0.8)$ \\
\hline Kankan & 29 & $1(3.5)$ & NA & NA & NA \\
\hline Kerouane & 67 & $1(1.5)$ & NA & NA & NA \\
\hline Kindia & 69 & $4(5.8)$ & NA & NA & NA \\
\hline Kissidougou & 94 & $1(1.1)$ & NA & NA & NA \\
\hline Macenta & 133 & $5(3.8)$ & NA & NA & NA \\
\hline N'Zérékoré & 101 & $1(1.0)$ & NA & NA & NA \\
\hline Telimele & 17 & 1 (5.9) & NA & NA & NA \\
\hline
\end{tabular}

Abbreviations: $\mathrm{NA}=$ not applicable; $\mathrm{VHF}=$ viral hemorrhagic fever.

* Twelve prefectures in which at least one alert call to the national call center was linked to a confirmed VHF case. Of the remaining prefectures, 11 had no confirmed VHF cases during the study period and 11 had at least one confirmed case, but no alert calls from the national call center were linked to VHF records (sensitivity $=0 \%$ ).

† November 5, 2014-August 31, 2015.

$\S$ April 1, 2015-August 31, 2015.

"Active prefectures are those with active Ebola cases during the period since the prefecture alert databases were standardized in Dubréka, Conakry, Coyah, and Forécariah prefectures on April 1, 2015; data before this date are incomplete and inconsistent across prefectures.

${ }^{1}$ Epidemic Intelligence Service, CDC; ${ }^{2}$ CDC Ebola Response Team, Conakry, Guinea; ${ }^{3}$ Division of Epidemiology, New York City Department of Health and Mental Hygiene; ${ }^{4}$ Naval Health Research Center, San Diego, California; ${ }^{5}$ Center for Global Health, CDC; ${ }^{6} \mathrm{CDC}$ Guinea Office, Conakry, Guinea.

Corresponding author: Christopher Lee, klq5@cdc.gov, 347-396-4070.

\section{References}

1. Baize S, Pannetier D, Oestereich L, et al. Emergence of Zaire Ebola virus disease in Guinea. N Engl J Med 2014;371:1418-25. http://dx.doi. org/10.1056/NEJMoa1404505

2. Kennedy M. WHO declares Ebola outbreak an international public health emergency. The Guardian. August 8, 2014. http://www.theguardian.com/society/2014/aug/08/ who-ebola-outbreak-international-public-health-emergency

3. World Health Organization. Ebola situation report-2 March 2016. Geneva, Switzerland: World Health Organization; 2016. http://apps. who.int/ebola/current-situation/ebola-situation-report-2-March-2016
4. Dixon MG, Schafer IJ. Ebola viral disease outbreak-West Africa, 2014. MMWR Morb Mortal Wkly Rep 2014;63:548-51.

5. Mortimer JY, Salathiel JA. 'Soundex' codes of surnames provide confidentiality and accuracy in a national HIV database. Commun Dis Rep CDR Rev 1995;5:R183-6.

6. Blasnik M. RECLINK: Stata module to probabilistically match records. Statistical Software Components from Boston College Department of Economics. 2010. http://econpapers.repec.org/software/bocbocode/ s456876.htm

7. Frieden TR, Damon I, Bell BP, Kenyon T, Nichol S. Ebola 2014—new challenges, new global response and responsibility. N Engl J Med 2014;371:1177-80. http://dx.doi.org/10.1056/NEJMp1409903

8. Miller LA, Stanger E, Senesi RG, et al. Use of a nationwide call center for Ebola response and monitoring during a 3-day house-to-house campaign-Sierra Leone, September 2014. MMWR Morb Mortal Wkly Rep 2015;64:28-9. 